\title{
Novel Strategies Reflect the Paradigm Shift in the Treatment of Heart Failure with Reduced Ejection Fraction
}

\begin{abstract}
Keisuke Kida
Key words: angiotensin receptor neprilysin inhibitor, blood pressure, diuretics, heart failure with reduced ejection fraction, renal function, strategies
\end{abstract}

(Intern Med 60: 2709-2710, 2021)

(DOI: 10.2169/internalmedicine.7288-21)

Novel strategies reflect the paradigm shift in the treatment of heart failure with reduced ejection fraction (HFrEF) that has occurred in recent years. In Japan, Ivabradine, a selective inhibitor of the If channel that purely reduces heart rate (HR) (1), sacubitril/valsartan and selective sodium-glucose cotransporter 2 (SGLT2) inhibitor were approved in 2019 and 2020, respectively. Sacubitril/valsartan is the combination of the neprilysin inhibitor pro-drug sacubitril and valsartan, an angiotensin II type 1 receptor blocker (ARB) in a new class of drugs called angiotensin receptor neprilysin inhibitor (ARNI). In the 5 years since the publication of the results of PARADIGM-HF (2), further insight has been gained into integrating a neprilysin inhibitor into a comprehensive multidrug regimen, including a renin-angiotensin aldosterone system blocker (3). Expert consensus recommends monitoring of blood pressure, potassium, and renal function after initiation and during titration in HFrEF patients taking ARNI as well as angiotensin-converting enzyme inhibitor (ACEI)/ARB (4).

Imamura et al. published a case report in which they described the switching from ACEI to ARNI during hospitalization in a patient with HFrEF and chronic kidney disease (CKD) (5). To reduce the risk of angioedema, a wash-out period of 36 hours was applied between the last dose of ACEI and initiation of ARNI. Because the HR was above 75 bpm in sinus rhythm in the setting of intolerance to the further up-titration of beta blocker due to dizziness on day 5, the systolic blood pressure (SBP) remained $120-130 \mathrm{mmHg}$ although ivabradine was considered as option. The authors initiated ARNI and slightly decreased down to 110-120 $\mathrm{mmHg}$ without symptomatic hypotension. Following the administration of ARNI, HR also decreased from $83 \mathrm{bpm}$ to under $75 \mathrm{bpm}$, indicating an improvement in heart failure without ivabradine administration.

In a TITRATION trial, a large majority $(-80 \%)$ of patients with low screening SBP levels $(100-110 \mathrm{mmHg}$ and 111$120 \mathrm{mmHg}$ ) were able to achieve and tolerate the target dose of ARNI using a gradual up-titration regimen (6). These findings suggest that low SBP should not prevent clinicians from considering the initiation of ARNI. However, we need to be careful in interpreting that not only the highest incidences of hypotension-related adverse events and SBP of $<95 \mathrm{mmHg}(21 \%)$ occurred among patients in the lowest SBP category $(100-110 \mathrm{mmHg}$ ), but also that the baseline characteristics of study participants such as age (61 years old), gender ( $81 \%$ male), and body size (BMI $30 \mathrm{~kg}$ / $\left.\mathrm{m}^{2}\right)$.

Treatment with ARNI was associated with more loop diuretic dose reductions and fewer dose increases compared with ACEI in the PARADIGM-HF study, suggesting that treatment with ARNI may reduce the relative requirement for loop diuretics in patients with HFrEF (7). The use of loop diuretics suggests a high risk of hypotension, electrolyte abnormalities and worsening renal function. Surprisingly, the authors were able to terminate loop diuretics during hospitalization (5). ARNI seems to be "diuresis-sparing" and loop diuretic dose may need to be reduced during or after up-titration. Compared with ACEI, ARNI also led to a slower rate of decrease in the estimated glomerular filtration rate and improved renal and cardiovascular outcomes, even in patients with HFrEF and CKD (8).

Finally, the patient was discharged with a simple and effective regimen of ARNI, beta-blocker and mineralocorticoid receptor antagonist on day 10 (5). In addition to these drugs, therapeutic strategies using SGLT2 inhibitors are gaining attention, and a new sequencing algorithm includes three 
steps, starting with HFrEF patients in whom loop diuretics have achieved clinical euvolemia (9). The arrival of such new drugs marks the beginning of a new era in developing optimal treatment strategies for HFrEF in Japan.

\section{Author's disclosure of potential Conflicts of Interest (COI).}

Keisuke Kida: Honoraria, Novartis Pharmaceuticals, Otsuka Pharmaceutical, Astrazeneca and Ono Pharmaceutical.

\section{References}

1. Tsutsui H, Momomura SI, Yamashina A, et al. Efficacy and safety of ivabradine in Japanese patients with chronic heart failure JSHIFT study. Circ J 83: 2049-2060, 2019.

2. McMurray JJ, Packer M, Desai AS, et al. Angiotensin-neprilysin inhibition versus enalapril in heart failure. $\mathrm{N}$ Engl $\mathrm{J}$ Med 371: 993-1004, 2014.

3. Docherty KF, Vaduganathan M, Solomon SD, et al. Sacubitril/valsartan: neprilysin inhibition 5 years after PARADIGM-HF. JACC Heart Fail 8: 800-810, 2020.

4. Writing Committee, Maddox TM, Januzzi JL Jr, et al. 2021 Update to the 2017 ACC Expert Consensus Decision Pathway for Optimization of Heart Failure Treatment: Answers to 10 Pivotal Issues About Heart Failure With Reduced Ejection Fraction: A Re- port of the American College of Cardiology Solution Set Oversight Committee. J Am Coll Cardiol 77: 772-810, 2021.

5. Imamura T, Hori M, Kinugawa K. Optimal therapeutic strategy using sacubitril/valsartan in a patient with systolic heart failure and chronic kidney disease -an initial case report in Japan. Intern Med 60: 2807-2809, 2021.

6. Senni M, McMurray JJV, Wachter R, et al. Impact of systolic blood pressure on the safety and tolerability of initiating and uptitrating sacubitril/valsartan in patients with heart failure and reduced ejection fraction: insights from the TITRATION study. Eur J Heart Fail 20: 491-500, 2018.

7. Vardeny O, Claggett B, Kachadourian J, et al. Reduced loop diuretic use in patients taking sacubitril/valsartan compared with enalapril: the PARADIGM-HF trial. Eur J Heart Fail 21: 337-341, 2019.

8. Damman K, Gori M, Claggett B, et al. Renal effects and associated outcomes during angiotensin-neprilysin inhibition in heart failure. JACC Heart Fail 6: 489-498, 2018.

9. McMurray JJV, Packer M. How should we sequence the treatments for heart failure and a reduced ejection fraction? A redefinition of evidence-based medicine. Circulation 143: 875-877, 2021.

The Internal Medicine is an Open Access journal distributed under the Creative Commons Attribution-NonCommercial-NoDerivatives 4.0 International License. To view the details of this license, please visit (https://creativecommons.org/licenses/ by-nc-nd/4.0/).

(C) 2021 The Japanese Society of Internal Medicine Intern Med 60: 2709-2710, 2021 